PROCEEDINGS OF THE AMERICAN MATHEMATICAL SOCIETY

Volume 127, Number 3, March 1999, Pages 847-849

S 0002-9939(99)04528-1

\title{
A VARIANT OF THE DIAMOND PRINCIPLE FOR COMBINATORIAL IDEALS
}

\author{
Y. ABE \\ (Communicated by Andreas R. Blass)
}

\begin{abstract}
We use a variant of the diamond principle to show many ideals on $\kappa$ are not $2^{\kappa}$-saturated if $\kappa$ is large. For instance, the $\Pi_{1}^{1}$-indescribable ideal is not $2^{\kappa}$-saturated if $\kappa$ is almost ineffable.
\end{abstract}

Kunen proved that the diamond principle for $\kappa, \diamond(\kappa)$ holds if $\kappa$ is subtle. A consequence of $\diamond(\kappa)$ is that the nonstationary ideal on $\kappa$ is not $2^{\kappa}$-saturated.

Meanwhile Baumgartner, Taylor and Wagon [2] proved that the ethereal ideal on $\kappa$ is not $\kappa^{+}$-saturated if $\kappa$ is almost ineffable.

These two facts have a point in common. If $\kappa$ has a strong property, then an ideal corresponding to a weaker property is less saturated.

For a regular uncountable cardinal $\kappa, \diamond(\kappa)$ can be regarded as a property of the nonstationary ideal. We consider the following principle for an ideal $I$ on $\kappa$ :

The Diamond Principle for $I, \diamond(I)$. There is a sequence $\left\langle S_{\alpha} \subset \alpha \mid \alpha<\kappa\right\rangle$ such that for every $X \subset \kappa$,

$$
\left\{\alpha<\kappa \mid X \cap \alpha=S_{\alpha}\right\} \notin I .
$$

We modify Kunen's construction of a diamond sequence assuming $\kappa$ has a sufficiently strong property so that $\diamond(I)$ holds. It is clear that no ideal $J \subseteq I$ is $2^{\kappa}$-saturated if $\diamond(I)$ holds. Specifically we prove the following.

Theorem. (1) If $\kappa$ is almost ineffable, then any ideal extended by the $\Pi_{1}^{1}$-indescribable ideal on $\kappa$ is not $2^{\kappa}$-saturated.

(2) If $\kappa$ is completely ineffable, then any ideal extended by the ineffable ideal on $\kappa$ is not $2^{\kappa}$-saturated.

Before proving the theorem we state the definition of these ideals. Throughout the rest of this paper, $\kappa$ is a regular uncountable cardinal and $I$ is a $\kappa$-complete ideal on $\kappa$. The filter dual to an ideal $I$ is denoted by $I^{*}$, and $I^{+}$is the set $\{X \subset \kappa \mid X \notin I\}$.

Definition. Let $X \subset \kappa$.

(i) $X$ is $\Pi_{1}^{1}$-indescribable if for any $R \subset V_{\kappa}$ and $\Pi_{1}^{1}$ sentence $\varphi$ such that $\left\langle V_{\kappa}, \in, R\right\rangle \models \varphi$, there is $\alpha \in X$ such that $\left\langle V_{\alpha}, \in, R \cap V_{\alpha}\right\rangle \models \varphi$.

(ii) $X$ is almost ineffable if for any sequence $\left\langle S_{\alpha} \subset \alpha \mid \alpha<\kappa\right\rangle$ there is $S \subset \kappa$ such that $\left\{\alpha \in X \mid S_{\alpha}=S \cap \alpha\right\}$ is unbounded in $\kappa$.

Received by the editors October 9, 1996 and, in revised form, June 5, 1997.

1991 Mathematics Subject Classification. Primary 03E05, 03 E55.

Key words and phrases. The diamond principle, saturated ideals, ineffability, indescribability.

(C)1999 American Mathematical Society 
(iii) $X$ is ineffable if for any sequence $\left\langle S_{\alpha} \subset \alpha \mid \alpha<\kappa\right\rangle$ there is $S \subset \kappa$ such that $\left\{\alpha \in X \mid S_{\alpha}=S \cap \alpha\right\}$ is stationary in $\kappa$.

(iv) The completely ineffable ideal on $\kappa$ is the minimal normal ideal $I$ such that for any $X \in I^{+}$and any sequence $\left\langle S_{\alpha} \subset \alpha \mid \alpha<\kappa\right\rangle$ there is $S \subset \kappa$ such that $\left\{\alpha \in X \mid S_{\alpha}=S \cap \alpha\right\} \in I^{+} . X \in I^{+}$is called completely ineffable.

(v) $X$ is subtle if for any sequence $\left\langle S_{\alpha} \subset \alpha \mid \alpha<\kappa\right\rangle$ and $C$ closed unbounded in $\kappa$, there exist $\alpha<\beta$ both in $C \cap X$ such that $S_{\alpha}=S_{\beta} \cap \alpha$.

For each property $A$ stated above, we consider the set

$$
\{X \subset \kappa \mid X \text { does not have property } A\},
$$

which is a normal ideal on $\kappa$. For instance the $\Pi_{1}^{1}$-indescribable ideal is the set

$$
\left\{X \subset \kappa \mid X \text { is not } \Pi_{1}^{1} \text {-indescribable }\right\} \text {. }
$$

These ideals were studied in Baumgartner [1] and Johnson [4].

Proof of the Theorem. (1) Suppose that $\kappa$ is almost ineffable. Let $N A I n_{\kappa}$ denote the almost ineffable ideal on $\kappa$ and $P_{\alpha}$ the $\Pi_{1}^{1}$-indescribable ideal on $\alpha$ for $\alpha \leq \kappa$. We use the fact that $P_{\kappa} \subset N A I n_{\kappa}$ and for every $X \in P_{\kappa}^{*}$,

$$
\left\{\alpha \in X \mid X \cap \alpha \in P_{\alpha}^{*}\right\} \in N A I n_{\kappa}^{*} .
$$

We recursively define $\left(S_{\alpha}, C_{\alpha}\right)$ for $\alpha<\kappa$ such that $S_{\alpha} \subset \alpha$ and $C_{\alpha} \in P_{\alpha}^{*}$ as follows.

Suppose that $\alpha<\kappa$ and $\left(S_{\beta}, C_{\beta}\right)$ has been defined for $\beta<\alpha$. Set $\left(S_{\alpha}, C_{\alpha}\right)=$ $(\emptyset, \alpha)$ except in the case that

$(\varnothing)$ : There exist $S \subset \alpha$ and $C \in P_{\alpha}^{*}$ such that $S \cap \beta \neq S_{\beta}$ for any $\beta \in C$.

In this case, let $\left(S_{\alpha}, C_{\alpha}\right)$ be one such pair $(S, C)$.

We show that $\left\langle S_{\alpha} \mid \alpha<\kappa\right\rangle$ is a diamond sequence for $P_{\kappa}$. Suppose to the contrary that there are $X \subset \kappa$ and $C \in P_{\kappa}^{*}$ such that $X \cap \alpha \neq S_{\alpha}$ for $\alpha \in C$. Let $D=\left\{\alpha \in C \mid C \cap \alpha \in P_{\alpha}^{*}\right\}$. For $\alpha \in D,(S \cap \alpha, C \cap \alpha)$ satisfies the condition of (囚). Hence $C_{\alpha} \in P_{\alpha}^{*}$ and $S_{\alpha} \cap \beta \neq S_{\beta}$ for $\beta \in C_{\alpha}$. Since $D \in N A I n_{\kappa}^{*}$, D is subtle. By Theorem 4.1 in Baumgartner [1],

$$
\left\{\alpha \in D \mid\left\{\beta \in D \cap \alpha \mid S_{\beta} \neq S_{\alpha} \cap \beta\right\} \in P_{\alpha}\right\} \text { is not subtle. }
$$

Thus we have

$$
E=\left\{\alpha \in D \mid\left\{\beta \in D \cap \alpha \mid S_{\beta}=S_{\alpha} \cap \beta\right\} \in P_{\alpha}^{+}\right\} \in N A I n_{\kappa}^{*} .
$$

For any $\alpha \in E, C_{\alpha} \in P_{\alpha}^{*}$. Hence we can find $\beta \in C_{\alpha}$ such that $S_{\beta}=S_{\alpha} \cap \beta$ contradicting the definition of $\left(S_{\alpha}, C_{\alpha}\right)$.

(2) Suppose that $\kappa$ is completely ineffable. Let $N C I n_{\kappa}$ denote the completely ineffable ideal on $\kappa$ and $N n_{\alpha}$ the ineffable ideal on $\alpha$ for $\alpha \leq \kappa$. We need only replace $P_{\alpha}^{*}$ by $N I n_{\alpha}^{*}$ in the definition of $\left(S_{\alpha}, C_{\alpha}\right)$ to get a diamond sequence for $N I n_{\kappa}^{*}$.

Consider the notion of forcing $Q=\left(N C I n_{\kappa}^{+}, \subseteq\right)$ and let $G$ be a $V$ generic filter on $Q$ and $M=U l t_{G}(V)$ the generic ultrapower. Since $N C I n_{\kappa}$ is normal $(\kappa, \kappa)$ distributive, $V_{\kappa+1}^{V}=V_{\kappa+1}^{M}$. (See [3], [4].) Hence, $N I n_{\kappa}^{V}=N I n_{\kappa}^{M}$ and, for any $X \in N I n_{\kappa}^{*}$,

$$
\left\{\alpha \in X \mid X \cap \alpha \in N \operatorname{In}_{\alpha}^{*}\right\} \in N C \operatorname{In}_{\kappa}^{*} .
$$


If $\left\langle S_{\alpha} \mid \alpha<\kappa\right\rangle$ is not a diamond sequence for $N I n_{\kappa}$, there is $Y \in N I n_{\kappa}^{*} \subset N C I n_{\kappa}^{*}$ such that, for any $\alpha \in Y$,

$$
C_{\alpha} \in N \text { In }_{\alpha}^{*} \text { and } S_{\beta} \neq S_{\alpha} \cap \beta \text { for } \beta \in C_{\alpha} .
$$

By complete ineffability, there exist $T, U \subset \kappa$ such that

$$
H=\left\{\beta \in Y \mid S_{\alpha}=T \cap \alpha \text { and } C_{\alpha}=U \cap \alpha\right\} \in N C \operatorname{In}_{\kappa}^{+} .
$$

Since $H \Vdash U \in N I n_{\kappa}^{*}, U \cap H \in N I n_{\kappa}^{+}$. For any $\beta<\alpha$ both in $U \cap H, \beta \in$ $U \cap \alpha=C_{\alpha}$ and $S_{\beta}=T \cap \beta=(T \cap \alpha) \cap \beta=S_{\alpha} \cap \beta$, which contradicts the fact that $\alpha \in Y$.

There are several facts which can be proved by the same argument. For instance:

If $\kappa$ is ineffable, then the $\Pi_{2}^{1}$-indescribable ideal on $\kappa$ is not $2^{\kappa}$-saturated.

If $\kappa$ is 2-subtle, then the ineffable ideal on $\kappa$ is not $2^{\kappa}$-saturated.

If $\kappa$ is measurable, then the completely ineffable ideal on $\kappa$ is not $2^{\kappa}$-saturated.

Such an argument can be carried out for ideals on $P_{\kappa} \lambda$ as well.

Johnson proved in [4] that the completely ineffable ideal is not precipitous if $\kappa$ is completely ineffable. Thus it seems natural to ask:

Question. (1) Can it be proved that these combinatorial ideals mentioned above are not precipitous?

(2) Is it possible to prove the ideal corresponding to property $A$ is not $2^{\kappa}$-saturated just assuming $\kappa$ has property $A$ ? For instance, in order to prove the ineffable ideal on $\kappa$ is not $2^{\kappa}$-saturated, does it suffice to assume $\kappa$ is ineffable?

\section{REFERENCES}

[1] J. Baumgartner, Ineffability properties of cardinals 1, Infinite and finite sets (P. Erdös 60th Birthday Colloquium, Keszthely, Hungary, 1973), Colloquia Mathematica Societatis János Bolyai, vol. 10, North-Holland, Amsterdam (1975), 109-130. MR 52:5427

[2] J. Baumgartner, A. Taylor and S. Wagon, On splitting stationary subsets of large cardinals, J. Symbolic Logic 42 (1977), 203-214. MR 58:21619

[3] C. A. Johnson, Distributive ideals and partition relations, J. Symbolic Logic 51 (1986), 617625. MR 87j:03076

[4] C. A. Johnson, More on distributive ideals, Fund. Math. 128 (1987), 113-130. MR 89a:03095

Department of Mathematics, Kanagawa University, Yokohama 221, Japan

E-mail address: yabe@cc.kanagawa-u.ac.jp 